\title{
How to be happy in general practice: a personal view
}

Thirty-four years in the same room in my practice at the end of the High Street; I closed the surgery door for the last time: I cried on the way home. Now after 15 years of wonderful retirement I reflect on what were the elements of my job that made me so happy through my working life.

First and foremost I had a 'personal' list in a group practice: three or four thousand diverse people who across the years brought to me a pot-pourri of problems from the trivial to the tragic. I observed every conceivable emotion, every type of human behaviour and a comprehensive catalogue of clinical illness. Not surprising then, that after 34 years of responding to these people they became almost friends. (Well, 300 people turned up for my 'leaving party' with an embarrassment of gifts!). With a large list the clinical volume of the commoner conditions was considerable, hence expertise was maintained, and even rarer conditions cropped up fairly frequently (I had four patients with Addison's Disease!). With careful organisation of appointment systems, and referral back from colleagues, a personal list did not necessitate burdensome availability. I saw all my patients' major problems and about $80 \%$ of their minor ones. Hence I countered the frequently expressed criticism that people 'never see the same person' and that there is poor continuity of care. And being one of a group of five doctors provided the medical camaraderie (and conflict!) to prevent medical isolation.

I worked from well-equipped self-owned premises and we had a major extension or refurbishment about every 10 years. We were quite proud of the old place!

I was a member of a multidisciplinary team. Many of us met daily, others less frequently, but usually at known times. Nurses, midwives, health visitors, social workers, counsellors, dieticians, and physiotherapists supplemented my clinical care and attended to many of the psychosocial problems presenting as 'disease'. Nurses in particular expanded their role and their numbers increased across the years. Preventative care was largely done by them. For chronic clinical illness they worked to practice-ordained protocols.

'Homely comforts' were important for all team members. We employed a 'tea lady' to serve quality tea and coffee twice daily in the team common room and all members were happy to attend!

We delegated administration, paper and computer work, recording and summarising, data collection and statistics to a large lay staff, employable as a result of the income generated by the large lists. It was gratifying to see how conscientiously the staff undertook these tasks - what could be tedious for doctors proved challenging and interesting to them. From the data and statistics flowed many papers and several books. This gave the whole team a 'buzz'.

Quality clinical care was the bedrock of our practice. Teaching and learning from trainees was helpful in this. We ordained a half-day per week for 'study', a monthly team clinical meeting, and attended a week-long 'refresher course' once a year. Six-month sabbaticals were undertaken by some of us including visiting professorships to Canada and the US and two practice exchanges with Canadian colleagues. Ennui and 'burn-out' was avoided.

Overall I tried to diagnose and treat patients without extensive investigations and referral to hospital. I examined patients repeatedly. I minimised medication and had low prescribing costs. Latterly, individual doctors developed 'special interests' - diabetes, skin, asthma, minor surgery, family planning - all collaborating with nurses. Cross referral within the practice began and we reckoned to reduce hospital referrals further - the expensive part of the NHS.

As in virtually every practice 'minor illness' was the volume problem. However, its presentation provided an opportunity for a quick review of 'preventative care' procedures that may not have been done. Arrangements for them could be instituted, we did not aggrandise it and self-care was encouraged. Truly minor illness received a minor response! Specially trained nurses shared it as there was enough to go around.

Home visiting, once the 'jewel in the crown' of British general practice, took up far too much time. I saw and encouraged its demise in the 70s and 80s and it was largely replaced by telephone consultations. But selectively it continued to be one of the most rewarding aspects of the job and because of it, quite serious illness could be cared for at home. In addition, doctors and nurses visited the housebound disabled and chronic sick. We generally visited the newborn and occasionally patients discharged from hospital after major illness or drastic surgery. Of major importance were visits to the dying where we tried to provide quality care at home for (often) long cared for patients. Such events were not numerous but the visits bonded us to the caring families and were cherished and remembered by them. Most people want to die at home and the GP and his team are essential in facilitating this.

But most of these elements of the job are still attainable and I would recommend them to GPs who seek a happy working life. Perhaps those who are currently seeking to restructure future general practice might also bear them in mind.

\section{Geoffrey N Marsh}

DOI: 10.3399/bjgp09X420455 